\title{
Photoconductivity of Intrinsic Semiconductor Phthalocyanine- Based Covalent Organic Polymer with Benzimidazole Linkage
}

Received 00th January 20xx, Accepted 00th January 20xx

DOI: $10.1039 / \times 0 x \times 00000 x$
Ercan Duygulu, ${ }^{a}$ Onur Alev ${ }^{b}$ Yurii Chumakov ${ }^{b, c}$, Zafer Ziya Öztürk ${ }^{b}$, Mehmet Menaf Ayhan ${ }^{a *}$ and Fatma Yuksel ${ }^{\text {a* }}$
Novel phthalocyanine-based benzimidazole linked conjugated covalent organic polymer (NiPc-COP1) have been synthesized with multiple randomly ordered crystalline morphology. This unique alignment leads to significantly enhanced panchromatic lightabsorption capability, thus high photoconductivity with high photoresponce speed.

The photoconductive polymers are a class of functional solidstate materials which, can trigger significant photocurrent generation by the absorption of photons and have attracted substantial attention due to their successful application in optical and electronic devices. ${ }^{1-5}$ The design of efficient photoconductive polymers requires electron-rich intrinsic semiconductive and photoconductive building blocks with the modular assembly that allows the formation of multiple conducting pathways (through-bond and through-space charge transport). ${ }^{6}$ The through-bond charge transport can be reached with extended $\pi$-conjugated polymer with efficient charge delocalization whereas through space transport requires to form short long-range p-orbital overlap in the stacking direction. $2,7,8$ In that regards, the 2D polymers with columnar assemblies, so called 2D covalent organic framework (COF), has emerged as ideal platforms for constructing photoconductive devices since fulfil both descript criteria, but has the shortcoming of requiring elaborate structural design and considerable synthetic effort. ${ }^{9-15}$ Moreover, the studies also report that such design also increases photoresponsive speed of polymers compare to disordered $2 \mathrm{D} \pi$-conjugated polymers. $^{7}$

However, one should note that, while 2D columnar assembly increase conductivity of generated electricity due to additional conductive pathways, at the same time it causes a reduction of electric generation due to limited absorption of photons by

\footnotetext{
a. Department of Chemistry, Gebze Technical University, Gebze, 41400, Kocaeli, Turkey.E-mail:menafayhan@gtu.edu.tr, fatma@gtu.edu.tr

b. Department of Pyhsics, Gebze Technical University, Gebze, 41400, Kocaeli, Turkey c. Institute of Applied Physics, MD-2028, Chisinau, Moldova

Electronic Supplementary Information (ESI) available: [Experimental details and Fig. S1-12l. See DOI: 10.1039/x0xx00000x
}

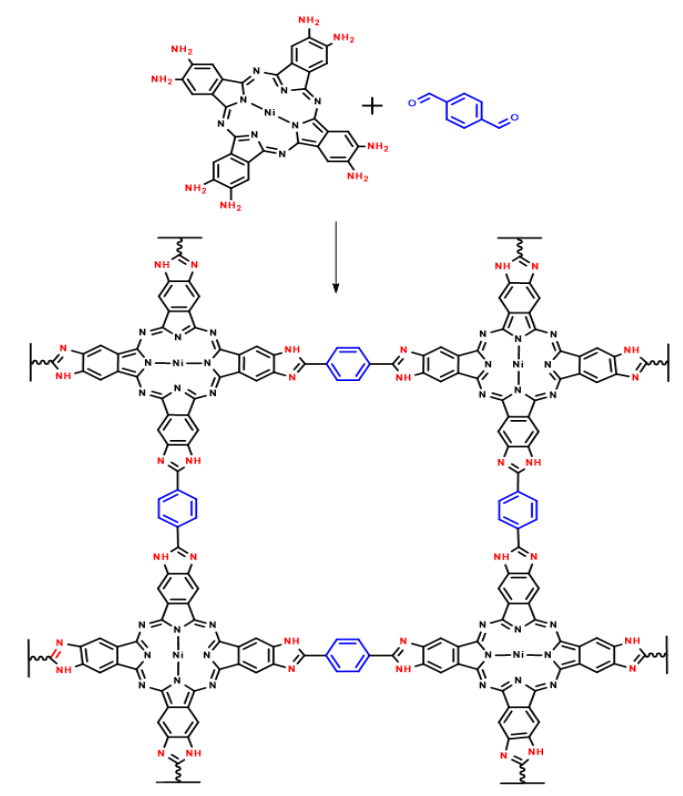

Scheme 1. Synthesis of NiPc-COP1

absorbents. ${ }^{16}$ On the other hand, 2D disordered $\pi$-conjugated polymers may have higher light absorption and consequently high electric generation but also would have low conductivity and low photoresponsive speed due to lack of additional conductive pathway. Thus, it is rather challenging to design photoconductive devices with high conductivity, high electricity generation and, let alone high photoresponsive speed. We hypothesized that a randomly ordered 2D fully $\pi-$ conjugated polymer could possess sufficient $\pi$-stacking to led to through space transport and necessary surface area to led strong light absorption to generate electricity. Consequently, device prepared based on this strategy could exhibit high intrinsic electrical generation and photoconductivity with high photoresponsive speed without considerable synthetic effort. The photoconductive polymers can be built upon the incorporation of highly electron-rich intrinsic semiconductive and photoconductive building blocks, such as pyrene, porphyrin and phthalocyanine (Pc), into the polymer backbone. $2,11,13-15,17-19$ 

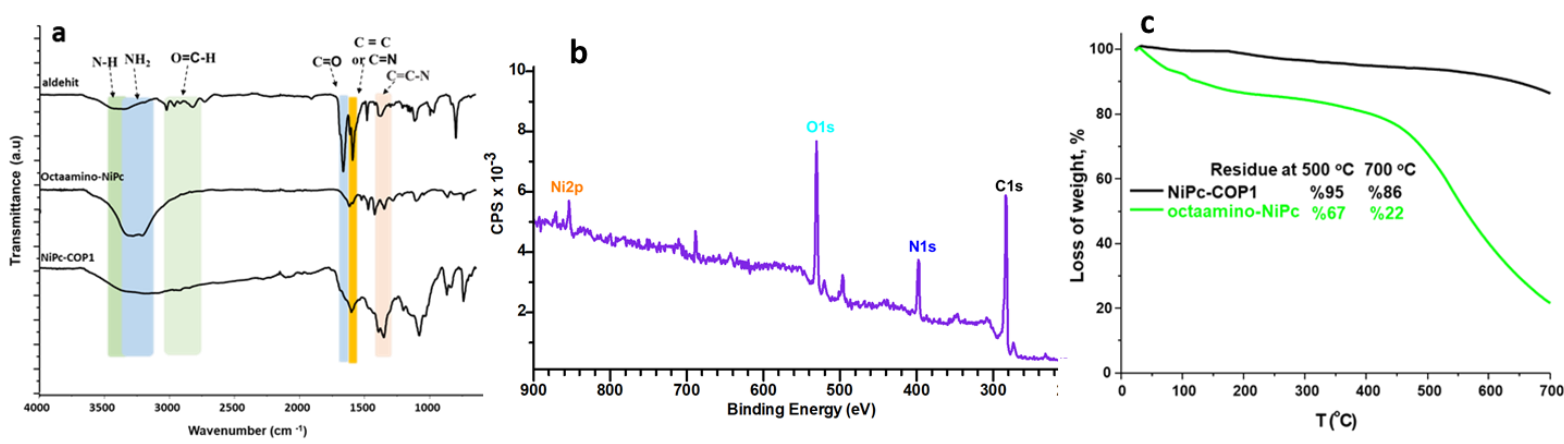

Figure 1. a) FT-IR of NiPc-COP1, octaaminoPc and 1,4 dialdehyde benzene. b) Survey scan XPS profiles of NiPc-COP1. c) TGA of NiPc-COP1 and octaaminoPc

Among these building blocks, Pcs are a class of planar aromatic organic compounds that have attracted considerable attention due to their unique properties such as semiconductivity, photoconductivity, photochemical reactivity, and chemical stability. ${ }^{12,20,29,30,21-28}$

To date, significant effort has been put into design and synthesis Pc-based devices with high conductivity. Most of the current strategies to obtain Pc-based polymers in literature are mainly based on dynamic covalent chemistry reactions such as, boronic acid condensation, boronate ester formation with octa-hydroxy Pc. ${ }^{10,13,18,20,31-34}$ These polymers are not conjugated yet possess moderate conductivity due to $\pi$ stacking induced conduction path. Lately conjugated and columnar stacked 2D Pc-COF based on imine formation were reported and has been proven to exhibit high intrinsic conductivity. ${ }^{27}$ However, examples of such high-performance conductive Pc-based structures are somewhat limited, so the further development of new polymers is a high priority.

Herein, we report the first example of benzimidazole linked phthalocyanine-based conjugated crystalline covalent organic polymer (NiPc-COP1, Scheme 1) and highlight its electrontransporting property and prominent photoconductivity.

NiPc-COP1 was synthesised as a dark green microcrystalline by condensation reaction of $\mathrm{Ni}$ (II) octa-amino phthalocyanine with 1,4-benzene-dialdehyde precursor under solvothermal condition as shown in Scheme 1 (The synthesis details are in ESI). Fourier-transform infrared (FT-IR) spectroscopy of NiPcCOP1 (Fig. 1a) showed the appearance of characteristic stretching bands of the imidazole system $(-\mathrm{NH},-\mathrm{C}=\mathrm{N})$ at 3329 and $1610 \mathrm{~cm}^{-1}$, while stretching bands of $\mathrm{C}=\mathrm{O}$ and $-\mathrm{NH}_{2}$ groups from octaamino-NiPc and 1,4-benzene-dialdehyde were absent, indicating the formation of benzimidazole linkages. The X-ray photoelectron spectroscopy (XPS) survey spectrum in Figure $1 \mathrm{~b}$, confirmed the presence of $\mathrm{C}, \mathrm{N}$, and $\mathrm{Ni}$ elements in NiPc-COP1. An unexpected $\mathrm{O}$ 1s peak might be due to the absorbed $\mathrm{H}_{2} \mathrm{O}$ molecule on the NiPc-COP1 surface. The highresolution scans of the $\mathrm{C} 1 \mathrm{~s}$ spectrum revealed emission lines with binding energies of 285.3, 286.5, and $288.7 \mathrm{eV}$, which were ascribed to the $\mathrm{C}=\mathrm{C}, \mathrm{C}=\mathrm{N}$, and $\pi-\pi^{*}$ species, respectively (Fig. S1). The study of N1s spectrum showed mainly three peaks at binding energies of 400, 401.5 and $402.9 \mathrm{eV}$, which were attributed to $\mathrm{C}-\mathrm{N}-\mathrm{H}, \mathrm{C}=\mathrm{N}-\mathrm{C}$ and $\mathrm{C}=\mathrm{N}-\mathrm{Ni}$, respectively. The existence of $\mathrm{C}-\mathrm{N}-\mathrm{H}$ peak and higher ratio $(65: 12)$ of the uncoordinated $\mathrm{C}=\mathrm{N}$ compared to the coordinated $\mathrm{C}=\mathrm{N}-\mathrm{Ni}$ indicated the formation of benzimidazole linkage during the NiPc-COP1 synthesis (Fig. S2). Furthermore, two peaks at 856.8
$\mathrm{eV}$ and $874.2 \mathrm{eV}$ are due to $\mathrm{Ni} 2 \mathrm{p} 3 / 2$ and $2 \mathrm{p} 1 / 2$, respectively and another satellite peak at $864.7 \mathrm{eV}$ indicates the existence of $\mathrm{Ni}^{2+}$ state in NiPc molecule, as shown in figure $\mathrm{S} 3.27,35,36$ Thermogravimetric analysis (TGA) shows that NiPc-COP1 exhibit excellent thermal stability up to $500^{\circ} \mathrm{C}$ with only slight weight losses (\%5) (Fig. 1c). Extremely stable structures lay a good foundation for further study in applications of NiPcCOP1.

Elemental mapping by energy dispersive spectroscopy (EDS) exhibits the elements $\mathrm{C}, \mathrm{N}$, and $\mathrm{Ni}$ (Fig. S4), which is consistent with the compositions of our NiPc-COP1. Scanning electron microscopy (SEM) studies reveal that exhibit a block-like morphology for NiPc-COP1 (Fig. S5). Dynamic light scattering (DLS) analyses showed two particle populations with an average colloidal particle size in the range between 1 and 10 $\mu \mathrm{m}$ for NiPc-COP1, suggesting a polydisperse nature (Fig. S6). The stability of colloidal systems of NiPc-COP1 was evaluated with zeta potential measurements in water $(\mathrm{pH}=7.4)$ (Fig. S7). The zeta potential of NiPc-COP1 shows a negative zeta potential value (-23.5) which indicate a stable state of their colloidal systems. The Powder X-ray diffractometry (PXRD) profile of NiPc-COP1 contains the low angle diffraction peak in vicinity of $2 \theta=4.5-6.0^{\circ}$ which is characteristic for expected large unit-cell parameters (Fig. S8). The experimental PXRD profiles of NiPc-COP1 revealed the formation of the multiple randomly ordered crystalline structures, probably due to multiple isomers of benzimidazole linkage with nearly equal total energy (Fig. S9, Table S1). NiPc-COP1 exhibited typical type-II reversible adsorption-desorption isotherm curves with a Brunauer-Emmett-Teller surface area of $23,9 \mathrm{~m}^{2} \mathrm{~g}^{-1}$ (Fig. S10). The pore-size distribution is found to be mesoporous in the range of 3-35 nm (Fig. S11). These relatively low values are likely due to randomly ordered morphology of NiPc-COP1.

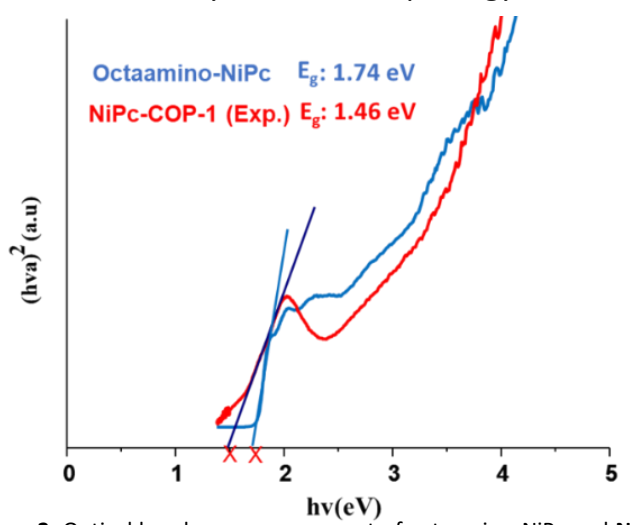

Figure 2. Optical band gap measurement of octaamino-NiPc and NiPc-COP1 by Tauc plotting of the DRS. 


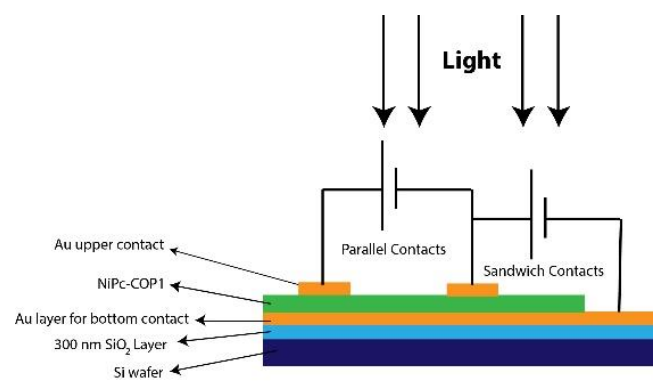

Figure 3. Contact formations for electrical and photoconductivity measurements.

The optical band gap of NiPc-COP1 in solid state has been studied with diffuse reflectance spectroscopy (DRS) using the relational expression proposed by Tauc, Davis, and Mott and calculation details are given in ESI. ${ }^{37-39}$ The Tauc plot derived from the first jump in the spectrum yields a narrow bandgap at ca. $1.46 \mathrm{eV}$ corresponding to the $\mathrm{S}_{0}-\mathrm{S}_{1}$ transition $\mathrm{Q}$ band of $\mathrm{Pc}$ core and shown in Figure 2. As expected, introducing the benzimidazole linkage into COP systems decrease the optical bandgap of NiPc-COP1 due to the extension of conjugation and enhances its capability to harvest light at lower energies. Moreover, the narrow bandgap of $1.46 \mathrm{eV}$, suggesting that NiPc-COP1 is a semiconductor.

To study the electrical and photoconductivity of NiPc-COP1, a film with a thickness of $100 \mathrm{~nm}$ was growth on $\mathrm{Au} / \mathrm{SiO} 2 / \mathrm{Si}$ substrates as described in ESI. The measurements were performed with two different electrode structures which are sandwich and parallel contact (Figure 3 ). The parallel contact electrode was used to determine electrical behaviour of surface of NiPc-COP1 whereas sandwich contact used for electrical behaviour of bulk system. The temperaturedependent electrical conductivity of NiPc-COP1 with a constant voltage of $10 \mathrm{~V}$ at a step rate of $1 \mathrm{~K}$ between $30 \mathrm{~K}$ and $300 \mathrm{~K}$ was performed. The conductivity of NiPc-COP1 increases almost linearly with increasing temperature (Fig. 4a), which indicates its semiconductive nature. The conductivity of NiPcCOP1 is found to be $2.978 \times 10^{-9}(\mathrm{~S} / \mathrm{cm})$ with $0.29 \mathrm{eV}$ the activation energy (for calculation see ESI) at room temperature. ${ }^{40-42}$ NiPc-COP1 based device exposed to light from a xenon light source $(\lambda=600 \mathrm{~nm})$ to investigate its photoconductivity. Interestingly, with the parallel contact electrode, device showed ohmic behavior and didn't exhibit photoconductivity, whereas with sandwiched contact, the device showed diode-like behavior and resulted in a significant increase in current from $2.7 \mu \mathrm{A}$ (dark current) to $4.5 \mu \mathrm{A}$ (photocurrent) with irradiation (Fig. 4b, 4c).

Furthermore, the wavelength dependence of the photosensitivity of NiPc-COP1 was also studied, the device was exposed with light passed through a monochromator with 50
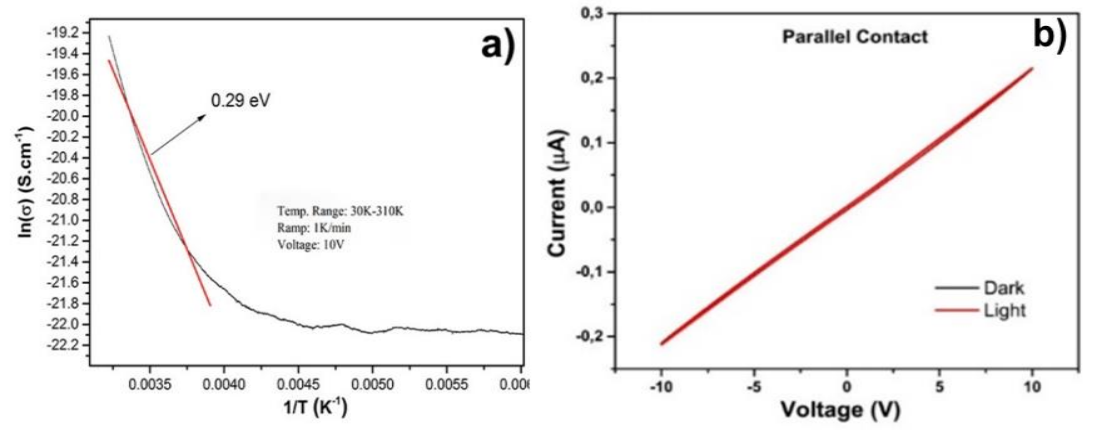

\section{Notes and references}

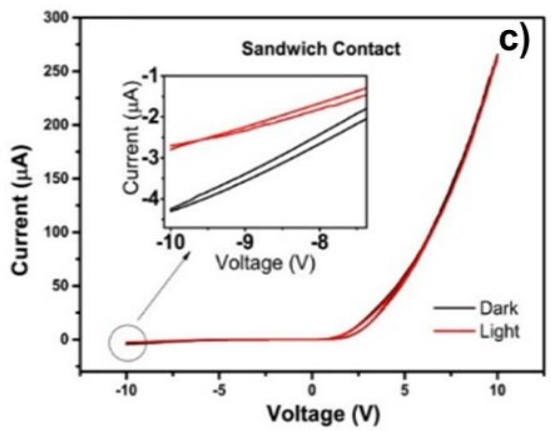

Figure 4. a) The dependence of logarithmic dc conductivity, In odc, on the inverse of the temperature, $1 / \mathrm{T}$, for NiPc-COP1. I-V curves of b) parallel contact and c) sandwich contact measurements under dark and light with $600 \mathrm{~nm}$ light. 

7, 1-8.

S. Wan, J. Guo, J. Kim, H. Ihee and D. Jiang, Angew. Chemie - Int. Ed., 2009, 48, 5439-5442.

H. Dong, H. Zhu, Q. Meng, X. Gong and W. Hu, Chem. Soc. Rev., 2012, 41, 1754-1808.

Y. Zhang, R. Tao, X. Zhao, Z. Sun, Y. Wang and L. Xu, Chem. Commun., 2016, 52, 3304-3307.

S. Reynolds, M. Brinza, M. L. Benkhedir and G. J.

Adriaenssens, 2017, 151-174.

E. Majda-Zdancewicz, M. Suproniuk, M. Pawłowski and M. Wierzbowski, Opto-electronics Rev., 2018, 26, 92-102. Y. Chen, H. Cui, J. Zhang, K. Zhao, D. Ding, J. Guo, L. Li, Z. Tian and Z. Tang, RSC Adv., 2015, 5, 92573-92576.

M. Souto and D. F. Perepichka, J. Mater. Chem. C, 2021, 9, 10668-10676.

A. Nath, K. S. Asha and S. Mandal, Chem. - A Eur. J., 2021, 27, 11482-11538.

E. Jin, K. Geng, S. Fu, S. Yang, N. Kanlayakan, M. A. Addicoat, N. Kungwan, J. Geurs, H. Xu, M. Bonn, H. I. Wang, J. Smet, T. Kowalczyk and D. Jiang, Chem, 2021, 1-16.

Y. Yue, P. Cai, X. Xu, H. Li, H. Chen, H. C. Zhou and N. Huang, Angew. Chemie - Int. Ed., 2021, 60, 10806-10813. M. Wang, M. Wang, H. H. Lin, M. Ballabio, H. Zhong, M. Bonn, S. Zhou, T. Heine, E. Cánovas, R. Dong and X. Feng, J. Am. Chem. Soc., 2020, 142, 21622-21627.

S. Jin, X. Ding, X. Feng, M. Supur, K. Furukawa, S. Takahashi, M. Addicoat, M. E. El-Khouly, T. Nakamura, S. Irle, S. Fukuzumi, A. Nagai and D. Jiang, Angew. Chemie - Int. Ed., 2013, 52, 2017-2021.

X. Feng, L. Liu, Y. Honsho, A. Saeki, S. Seki, S. Irle, Y. Dong, A. Nagai and D. Jiang, Angew. Chemie - Int. Ed., 2012, 51, 2618-2622.

S. Wan, F. Gándara, A. Asano, H. Furukawa, A. Saeki, S. K. Dey, L. Liao, M. W. Ambrogio, Y. Y. Botros, X. Duan, S. Seki, J. F. Stoddart and O. M. Yaghi, Chem. Mater., 2011, 23, 4094-4097.

A. W. Snow, in The Porphyrin Handbook, 2003, pp. 129176.

Y. Xiong, Q. Liao, Z. Huang, X. Huang, C. Ke, H. Zhu, C. Dong H. Wang, K. Xi, P. Zhan, F. Xu and Y. Lu, Adv. Mater., 2020, 32, 1-8.

X. Ding, J. Guo, X. Feng, Y. Honsho, J. Guo, S. Seki, P. Maitarad, A. Saeki, S. Nagase and D. Jiang, Angew. Chemie Int. Ed., 2011, 50, 1289-1293.

M. Lu, M. Zhang, C. G. Liu, J. Liu, L. J. Shang, M. Wang, J. N. Chang, S. L. Li and Y. Q. Lan, Angew. Chemie - Int. Ed., 2021 60, 4864-4871.

X. Ding, X. Feng, A. Saeki, S. Seki, A. Nagai and D. Jiang, Chem. Commun., 2012, 48, 8952-8954.

C. L. Yao, J. C. Li, W. Gao and Q. Jiang, Chem. - A Eur. J., 2018, 24, 11051-11058.

X. Ding, L. Chen, Y. Honsho, X. Feng, O. Saengsawang and J. Guo, 2011, 14510-14513.

L. Chen, K. Furukawa, J. Gao, A. Nagai, T. Nakamura, Y. Dong and D. Jiang, J. Am. Chem. Soc., 2014, 136, 98069809.

B. Han, X. Ding, B. Yu, H. Wu, W. Zhou, W. Liu, C. Wei, B. Chen, D. Qi, H. Wang, K. Wang, Y. Chen, B. Chen and J. Jiang, J. Am. Chem. Soc., 2021, 143, 7104-7113.

Z. Wang, L. S. Walter, M. Wang, P. S. Petkov, B. Liang, H. Qi, N. N. Nguyen, M. Hambsch, H. Zhong, M. Wang, S. Park, L. Renn, K. Watanabe, T. Taniguchi, S. C. B. Mannsfeld, T. Heine, U. Kaiser, S. Zhou, R. T. Weitz, X. Feng and R. Dong, J. Am. Chem. Soc., , DOI:10.1021/jacs.1c05051.

S. Jin, M. Supur, M. Addicoat, K. Furukawa, L. Chen, T. Nakamura, S. Fukuzumi, S. Irle and D. Jiang, J. Am. Chem. Soc., 2015, 137, 7817-7827.

Z. Meng, R. M. Stolz and K. A. Mirica, J. Am. Chem. Soc., 2019, 141, 11929-11937.

M. Di Zhang, D. H. Si, J. D. Yi, S. S. Zhao, Y. B. Huang and R. Cao, Small, 2020, 16, 1-8.

C. G. Claessens, U. Hahn and T. Torres, Chem. Rec., 2008, 8, 75-97.

F. W. Kutzler, W. R. Barger, A. W. Snow and H. Wohltjen, Thin Solid Films, 1987, 155, 1-16.

E. L. Spitler, J. W. Colson, F. J. Uribe-Romo, A. R. Woll, M. R. Giovino, A. Saldivar and W. R. Dichtel, Angew. Chemie - Int. Ed., 2012, 51, 2623-2627.

V. S. P. K. Neti, X. Wu, M. Hosseini, R. A. Bernal, S. Deng and L. Echegoyen, CrystEngComm, 2013, 15, 7157-7160. E. L. Spitler and W. R. Dichtel, Nat. Chem., 2010, 2, 672677.

X. Wang, M. Bahri, Z. Fu, M. A. Little, L. Liu, H. Niu, N. D. Browning, S. Y. Chong, L. Chen, J. W. Ward and A. I. Cooper, , DOI:10.1021/jacs.1c08351.

X. Wang, W. Wu, H. Ju, T. Zou, Z. Qiao, H. Gong and H. Wang, Mater. Res. Express, , DOI:10.1088/20531591/3/12/125002.

L. Ottaviano, S. Di Nardo, L. Lozzi, M. Passacantando, P. Picozzi and S. Santucci, Surf. Sci., 1997, 373, 318-332. J. Tauc, R. Grigorovici and A. Vancu, phys. stat. sol., 1966, 15, 625-637.

J. Tauc, Optical Properties of Solids, Abeles, North Holland, Amsterdam, 1972.

N. F. Mott and E. A. Davis, Philos. Mag., 1970, 22, 903-922. N. Kilinç, S. Öztürk, D. Atilla, A. G. Gürek, V. Ahsen and Z. Z. Öztürk, Sensors Actuators, B Chem., 2012, 173, 203-210. O. S. Josyulu and J. Sobhanadri, Phys. Status Solidi, 1980, 59, 323-329.

E. Kumral, H. Y. Yenilmez, S. Albayrak, A. N. Sąhin, A. Altindal and Z. A. Bayir, Dalt. Trans., 2020, 49, 9385-9392. B. Nath, W. H. Li, J. H. Huang, G. E. Wang, Z. H. Fu, M. S. Yao and G. Xu, CrystEngComm, 2016, 18, 4259-4263. 